\title{
A Creative Information System Based on the SCAMPER Technique
}

\author{
Rute Lopes ${ }^{1}$, Pedro Malta ${ }^{1}$, Henrique Mamede ${ }^{2}$, Vitor Santos ${ }^{1}$ \\ 1. NOVA IMS - Information Management School Universidade Nova de Lisboa, Lisbon \\ Portugal \\ 2. INESC TEC, Departamento de Ciências e TecnologiaUniversidade Aberta, Lisbon Portugal
}

This is the Author Peer Reviewed version of the following chapter published by Springer:

Lopes, R., Malta, P., Mamede, H., \& Santos, V. (2020). A Creative Information System Based on the SCAMPER Technique. In M. Themistocleous, M. Papadaki, \& M. M. Kamal (Eds.), Information Systems: 17th European, Mediterranean, and Middle Eastern Conference, EMCIS 2020, Proceedings (pp. 595-606). (Lecture Notes in Business Information Processing; Vol. 402). Springer Science and Business Media Deutschland $\mathrm{GmbH}$. https://doi.org/10.1007/978-3-030-63396-7_40

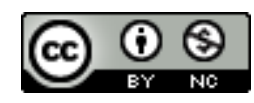

This work is licensed under a Creative Commons Attribution-NonCommercial 4.0 International License. 


\title{
A Creative Information System based on the SCAMPER Technique
}

\author{
Rute Lopes ${ }^{1}$, Pedro Malta ${ }^{1}$, Henrique Mamede $^{2}$ and Vitor Santos ${ }^{1}$ \\ ${ }^{1}$ NOVA IMS - Information Management School, Universidade Nova de Lisboa, \\ Lisboa - Portugal \\ \{m2015143; pmalta; vsantos\}@novaims.unl.pt; \\ 2 INESC TEC - Departamento de Ciências e Tecnologia - Universidade Aberta, Lisboa, \\ Portugal \\ hsmamedelgmail.com
}

\begin{abstract}
Nowadays, the use of creativity in business has been increasing drastically because it is important for the market to come up with new ways to find focused in answers to the problems proposed by the users. There are several different creativity techniques that can be used in different contexts. One of the most important techniques is the SCAMPER technique, which is based in reorganizing, modifying, adding and eliminating information. An automated system will provide answers and solutions to creativity problems and contribute to minimize the cost of in-novation in the companies. The aim of this thesis is therefore to design an architecture system for a creative information system based on the SCAMPER creativity technique making an auto-mated system of this technique.
\end{abstract}

Keywords: Creativity; Information Systems Architectures; SCAMPER; Creative Information Systems.

\section{Introduction}

For years now, analytical thinking has been at the basis of problem solving, where thinking is done by a recognition process and solutions are based on hypotheses, analogies or syntheses. These steps of vertical thinking can be accomplished not only by using data, but also by using creative thoughts [5].

There are a variety of definitions and ways to use creativity. Over the years, we have studied the impact and uses in everyday life and in business. The use of creativity techniques is a way to help improve and trigger creative thinking. In the early studies, creativity was quickly assumed to be an intrinsic characteristic of a person, although Guilford stated that the studies were inconclusive. Subsequently, the term design thinking emerges, which is the way and strategies of thinking, where the different styles and characteristics of the individual have a great role in the process of creating something new [1], [2].

It is expected that a person who is easier to approach design thinking 
will have a fluid, flexible and original thinking. These characteristics are the ones that are tested when creativity is in focus, but, anyway, the researchers have come to conclude that there is a need for a creative thinking approach when looking for new solutions [3], [4].

Many theories have been formulated around creativity, one of which is lateral thinking. Edward Bono described lateral thinking as a way of solving problems, moving from a known idea to a new one, based on standards and tools, as opposed to the traditional approach of finding solutions step by step [2]. Lateral thinking uses techniques that trigger people who are not so predisposed to creative thoughts and to promote creativity training in those who are, forcing the user to answer questions that would not normally come to mind and rearranging information into new patterns, resulting in into a problem and an opportunity [6], [7]

The scope of using these creative techniques is vast although they all have advantages and disadvantages and, depending on the situation can be useful.

The use of technology to recreate creativity using creativity techniques, where a system could help to increase creativity, giving different and original responses to different contexts, would be an important step to be taken in this field of study.

One good example of a successful technique is SCAMPER that reorganizes and combines in-formation to create different ideas according to a problem or situation. Created by Alex Osborn in 1953, it is one of the most complex techniques, because each letter of the acronym represents a different method of using [8].

These methods are: Substitute (materials, components, people), Combine (mix, combine, integrate), Adapt (change function), Modify Michalko added Magnify- (increase or decrease the scale, change shape), Put it to another use, Eliminate (remove, simplify, reduce) and Reverse - Michalko added Rearrange - (change components, change speed, turn inside out or upside down in order) [6].

The main advantage of the SCAMPER technique is that it promotes creative thinking when analyzing a problem and generates new ideas. A weakness of the SCAMPER technique is that it works only in limited environments, those that encourage free thinking, and the fact that the technique discourages group thinking because it is a non-group technique, although it is also recommended be carried out in a group, all discussions can lead to a dead end [6].

Is this paper we propose a system architecture for SCAMPER technique implementation. 


\section{Creative Information System}

In recent studies, a "Creative Information System" (CIS) was created, which is an automated system that produces autonomous responses, using a creative technique as an intellectual basis. It is important to note that there are minimum requirements for entries, namely the problem specification, its context and restrictions. Depending on the creativity technique, the system will generate the responses through the chosen process [9].

Therefore, this system can recreate the original technique with a minimum of human interaction: the system will receive an entry with the context and restrictions of the problem and will generate answers or solutions to the problem that can be analyzed later [10].

The model presented in Santos et al. (2008) state how to plan and design an architecture for CIS. In this model, the entry indicating the problem and the context must be specified by the user; then, the design is produced in two stages, the first representing the application of the creative technique and the second the generation of responses, resulting, ultimately, in the output for analysis.

This model was used to propose architectures for two different creative techniques: "White-board" and "Brute thinking". These techniques are also explained by Michalko and are based on the random association of words. The process goes through three steps, the first is the introduction of a word (the model uses the dictionary and the internet), the second step is the combination of the random word with the context provided and the final step is the list of phrases (con-text and random words) [11] - [13].

In the CIS architecture for the Whiteboard technique, the user input is the keywords using a random word combiner from the dictionary or the Internet. The results are a combination of keywords (context) and randomly generated words. The analysis of this list of combinations is done by the user and its objective is to know which ones are valid or not [11].

The system for the Brute Thinking technique goes through approximately the same process as that explained above. The main difference between the two models is that the architecture of brute thinking is a collaborative semi-automatic system (more than one user at the same time). The first entry is made by users with all the necessary context, this context will be used by the other participants to generate and register keywords that can describe and classify the context. The same happens with an initial word, after these two steps, the system can generate a random combination of words with the following structure: keyword, verb (randomly generated) and a characteristic of the initial word. [10]. 


\section{Methodology}

The research proposed here aims to develop an innovative Creative Information System architecture that draws from findings from two disciplines, namely IS research and creativity. It was established the claim that the Design Science Research (DSR) approach is ideal to pursue our interdisciplinary research effort. As defined in the literature, the DSR approach combines the construction and analysis of innovative artifacts aiming to expand knowledge on specific challenge-solving $[14,15]$.

The DSR methodology establishes a sequential research trajectory, beginning with the identification of a problem, followed by conceptualization and development of a challenge-solving artifact, and moving to the assessment and refinement of the latter. The objective is to expand knowledge of a specific problem domain via an iteration process that begins with a research question and then proceeds with its characteristic trajectory of construction, evaluation, and re-dressing of design artifacts [14]. The challenge-solving artifact developed in the DSR process in this paper is an architecture.

\section{A Creative Information System for SCAMPER}

As shown in figure 1, the process starts when the user enters the information in the system. This set of phrases passes through the SCAMPER generator, which is the part of the system that makes changes to the input using the three different lists as an external source for changes: list of verbs, list of subjects and list of materials.

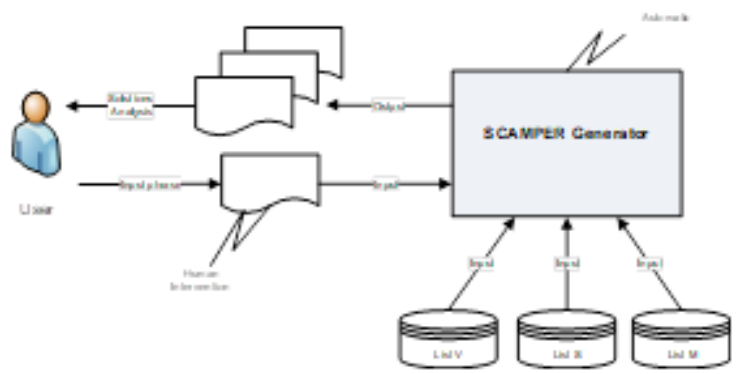

Fig. 1. CIS for the SCAMPER technique

The changes are documented in the output resulting in new sets of phrases in equal or higher number as the input phrases.

The input (figure 2) is produced by the user and has specific characteristics. It has a set of three or more phrases that combine four 
types of elements. The user can input as many sets of phrases as he wishes and can also leave in blank one or two of the phrases. However, in this last case only some of the methods of the SCAMPER technique can be used.

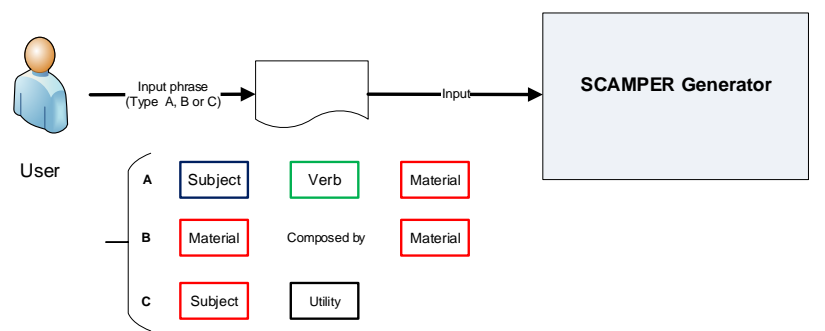

Fig. 2 - Type of input for the system

The phrase A combines a subject that can be a specific product or person/name, a verb which is an action or a composition and a material. The phrase B associates two, or more, types of materials, and the final phrase (phrase C) is a subject combined with an utility that can be a type of objects or a name of an object.

There are three types of lists that are an external mid-system input, which can be filled with words from a dictionary. List V represents the list of verbs and has two different columns. The first column contains all the necessary verbs and the second column has the opposite of the verbs in the first column. In this list, verbs can be repeated, as they can have more than one opposite and the same verb can be in both columns of the list. The subject is represented in the list $\mathrm{S}$ and contains only one column with the subject name. It can be a product, a person or a category. List $M$ consists of, in the first column, the name of the materials and in the second column the usefulness of the materials in column one. The materials can have more than one utility and vice versa, therefore, the materials and utilities can be repeated.

The SCAMPER generator works in different ways for each possible method, following various actions using the inputs explained above. It is important to realize that all the changes in the in-put are randomly made by the system.

The first method is "Substitution" and for that method the generator can use three different actions. These actions change directly two elements of the phrases, the materials and the subject, because the goal is to substitute materials to transform the final product and to change subject to transform the way a task is performed.

One of the actions in this method is to change the material in the phrase A for another of the list M. Changing the materials will give different ways to have the same product but with distinct characteristics. As represented in figure 3, using the list $\mathrm{M}$ the system will randomly choose the materials that differ from the one that is already in the phrase, and substitute that one for a material from the list. In this action 
the only focus is on the materials and they can be changed regardless of their utility.

The second action that we can list on the substitution method is also focused on the materials, but in this case uses the phrase B. As shown in the figure 3 , the system will go through the list of materials and choose randomly a material that is not the same that the system is changing. The action is performed as many times as materials of second instance exist as part of the phrases B. The purpose of this action is to have other materials to be part of the composition of the final solution. Again, the usefulness of the materials is not important for this step, as it provides only other options for existing materials.

The final action for the substitution method is focused on the subject in the A phrases; in this case, the system will search for subjects in List S and will randomly choose one to replace in the sentence that differs from the one that is already there (figure 3). This action intends to maintain the subject's objective, maintaining the verb and the material, but changes what or who performs it, changing the subject in order to find new ways to perform the task.

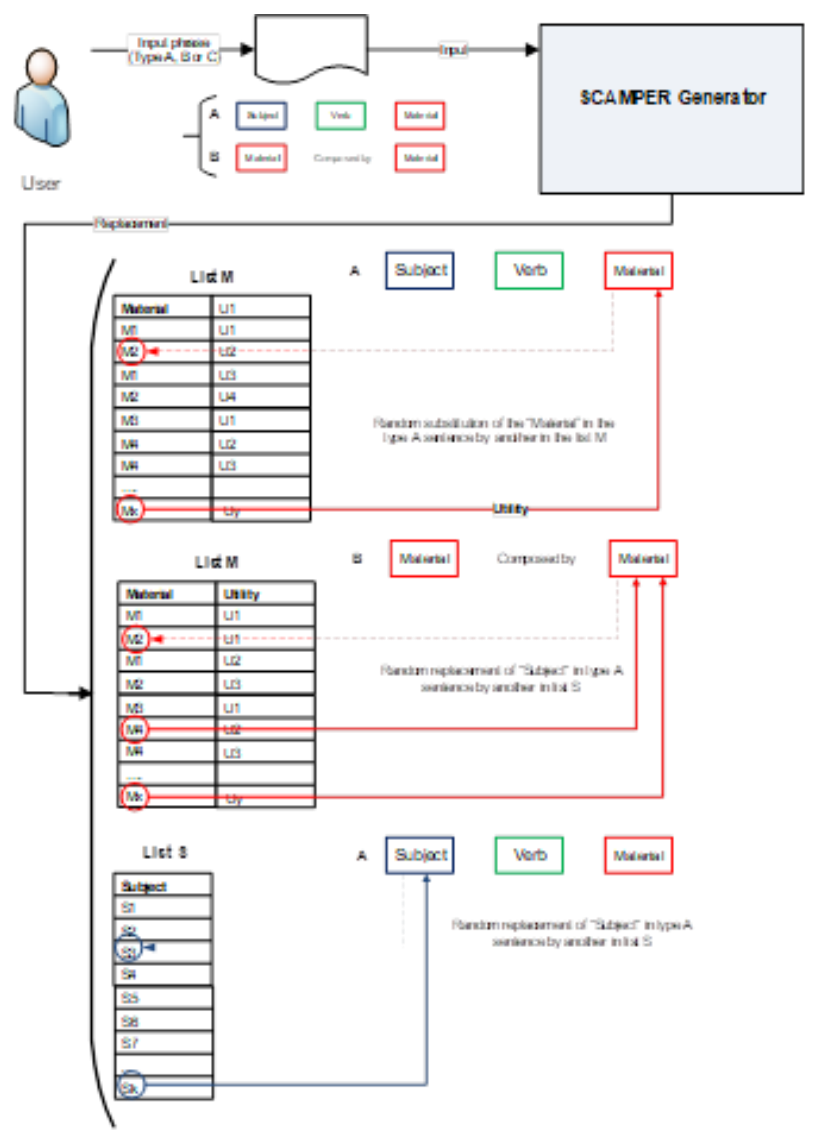

Fig. 3 - Actions for the Substitute method

The second method in the SCAMPER technique is "Combine". This method does not change the elements and instead combines different 
materials with the input of the phrases, as the goal is to add materials to transform the characteristics of the final product.

The system, as represented in figure 4, will go through the list of materials and choose randomly materials to add to the second instance of materials in sentence B. These materials are all different from those already included in the sentence in question. The number of materials to be add-ed is a random number between zero and five and can create the same combinations in different phrases or in all different combinations, as it is a random procedure. With this action, the SCAMPER generator will be able to supply different combinations of materials that may have the same utility or not; for this action, there is a focus on the different materials and not on their usefulness.

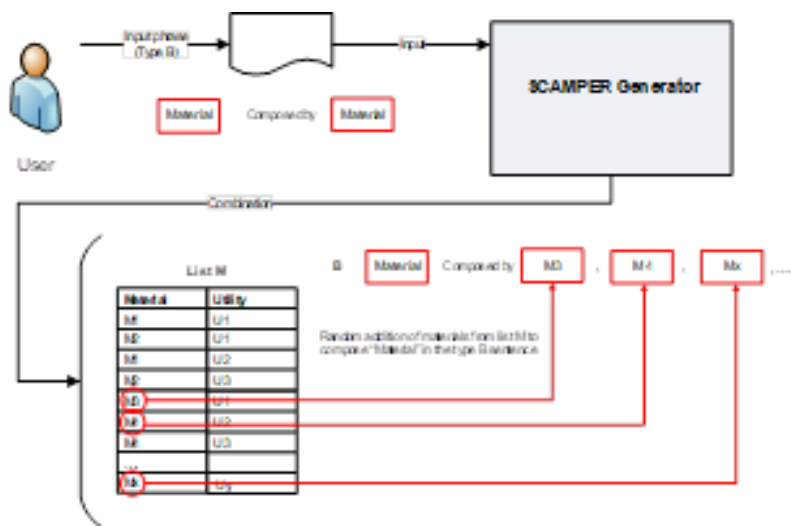

Fig. 4 - Action for the Combine method

Another SCAMPER method is "Adapt". In this case, the goal is to make changes to the materials or actions so that the initial product can be adapted to perform other actions or adapt materials to perform the actions of the initial product.

The first procedure used by the generator is related to the materials and verbs used in the entry in type A sentences. The objective is not to add or change the entry using the lists, but to adapt the materials and actions already used in sentences $A$ to other subjects. This procedure can only be performed by the system if there is a number of phrases A greater than one, as the system, as shown in figure 4, changes the materials and / or verbs in a set of phrases A.

The second action to consider is centered on type B phrases and materials. The aim is to change the composition of the products. The system can change the materials in one or more B phrases, so that the primary material becomes secondary and the same in reverse. As shown in figure 5 , the system will make these changes so that at the exit the materials are composed of different elements and, consequently, the solutions become more inventive. 
Another action that the system can take when using the adapt method is to join the materials of the type A and B phrases with other phrases of these two types of entry. The objective is again to change the materials in a way that theoretically adapts them to other actions and compositions.

The system will select some of the materials from phrases A and B and change them, so that a material that, for example, is part of the composition, becomes a main part of the product. In addition, materials in different type A phrases can be added to type B phrases without changing materials, keeping the material in phrase A. To perform this action, there must be at least one type of phrase A and one type of phrase $\mathrm{B}$ at the entrance.

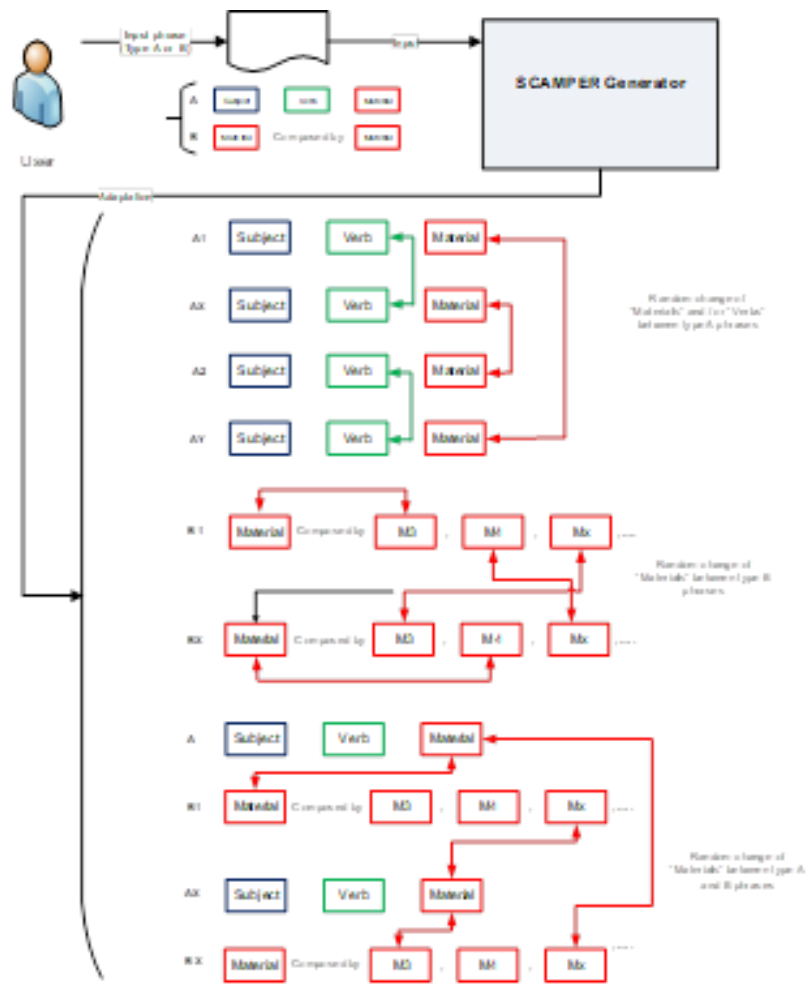

Fig. 5 - Actions for the Adapt method

The fourth method of the SCAMPER technique is "Modify". When using this method, changes are made to the materials, as the objective is to modify the attention that must fall on the useful-ness of the different materials. There is only one action to be taken with this method and it will change the materials in two different locations.

The action of this method is to randomly change the materials in sentence $\mathrm{A}$ and the materials of the second instance in sentence $\mathrm{B}$. The system, as represented in figure 6, will go through the list of materials (list M) and will randomly choose some materials to be replaced in phrases A and B. The system will identify materials that are different 
from those that are already in the entry of the phrases in question and that have the same utility as those that are in the entry. With this action, the SCAMPER generator will be able to supply different combinations of materials that can have the same utility; for this action, there is a focus on different materials that have the same utility.

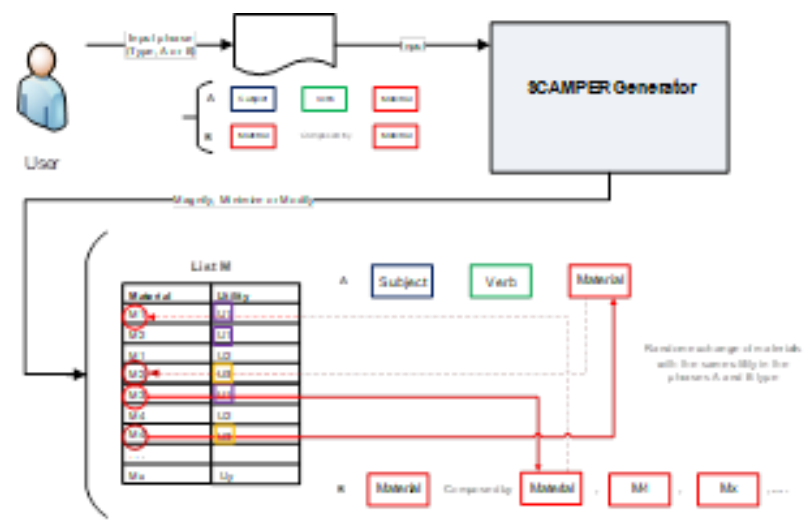

Fig. 6 - Actions for the Modify method

"Put to other use" is the next method in SCAMPER. There are two actions that can be performed on this method. In this case, the goal is to change the purpose of the entry, the actions it per-forms and the utility it has. The main changes will occur in the utility and in the verbs in the different types of sentences. These two actions will cause the output to change according to the essence of the method used for another use.

The first action is related to the verbs of sentence A. The objective is to change the action of the problem, placing the subject and the materials in different uses. The system, as represented in figure 7, will randomly search and find verbs in list $\mathrm{V}$ that are not the same as what is already in the input and will replace the input verb with these new verbs, creating new sentences. The only focus of this procedure is on verbs and they can be changed regardless of their opposite.

The second procedure of this method is in the usefulness of the different materials, the objective is to change the utility so that it can give new ways to use the materials. The system, as shown in figure 7 , will go through the first column of the list of materials and find the same material that is in the entry of sentence $C$, substituting in the sentence for the usefulness of the materials found, without repeating what is already there. The focus of this action is on utility and materials, as the system is looking for new uses for this material. 


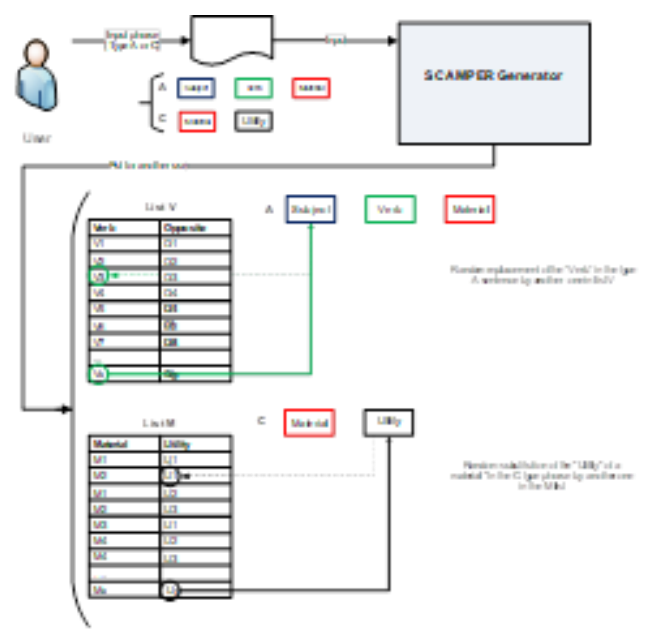

Fig. 7 - Actions for the Put to Other Use method

The next SCAMPER technique method is "Eliminate". There are two actions for the system to perform within this method. These actions have an impact on all elements of the system, regard-less of their origin and according to the essence of the method "eliminate". The goal is to eliminate parts of the input so that, in the end, the output becomes something different in terms of materials, action, utility or subjects.

The purpose of the first action of this method is to change the composition of the different products, so that the system eliminates the materials of phrase B. The rules for this elimination are: phrase B must have more than one material of second instance at the entrance; it can eliminate a random number between one and the number of second instance materials minus one, so you cannot delete all materials from the second instance. The system, represented in figure 8, will go through all the B phrases at the entrance and eliminate several materials of second in-stance; the materials will also be disposed of in random order.

In the second action, the system will need to modify the type A sentences and, consequently, the subject, verb and materials. The objective is to eliminate parts of the entry, so that the result of the system becomes different in different situations. To achieve this goal, the system, in figure 8, will recognize the A phrases and eliminate a set of arbitrary phrases, in a random number. The rules for this procedure are: the system can never delete all type A sentences; the number of sentences to be deleted is small and depends on how many type A sentences the entry has, so the system will eliminate several sentences between one and the total A phrases of the entry minus 1 . 


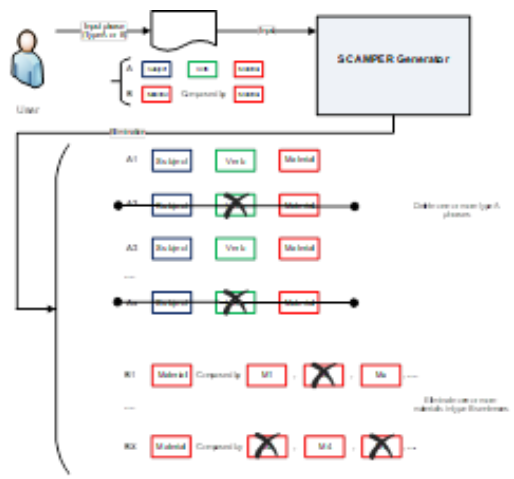

Fig. 8 - Actions for the Eliminate method

The last method in the SCAMPER technique is "Reverse". As the name demonstrates, the objective is to transform the entry into its opposite. In the system, there is only one action to be per-formed using this method; this action will manipulate the verbs, changing them. The goal is to replace the verbs to transform the actions that the final product produces, changing the main task to be performed.

The procedure in this final method is to change the verb in type A sentences by the opposite found in the verb list. The system, in figure 9 , will identify the verb in phrases A and look for the same verb in the column of verbs in list $\mathrm{V}$ and identify its opposite. The SCAMPER generator will replace the verbs in sentence A with the opposites found in the opposite column in list V. The purpose of this action is to cancel the action in the original entry and transform it into a different result, comparing it with the action performed in the SCAMPER technique.

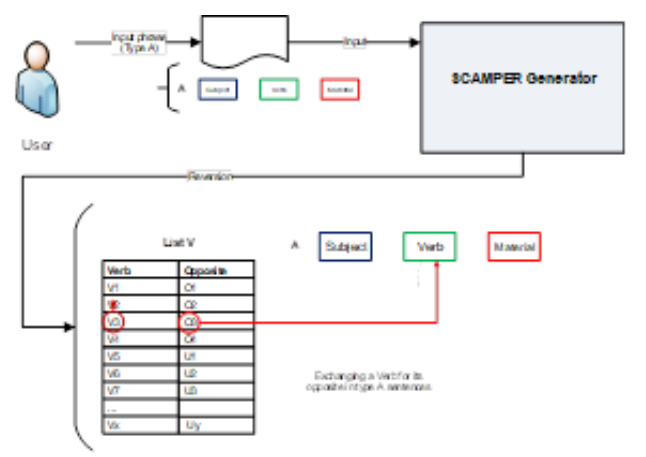

Fig. 9 - Action for the reverse method

The output is organized by sets of phrases with the same structure as the input. For example, if the user types ten phrases, the output will be a selected number, greater than one, from a set of phrases with several phrases that have been changed by the system.

The number of sentences sets in the output is chosen by the user at the input to facilitate the analysis of the responses. Answers are provided in sets of phrases and the number of sets is the result of the number of times the system makes separate changes to the entry. This way, the 
user will receive the number of possible outputs for him to analyses. It is important to note that the system may not provide valid responses to the problem, since what the system does is to provide several random responses that will later be analyzed by the user. As shown, the system can generate valid or invalid responses. Valid responses can be used as outputs from the system for further analysis.

\section{Discussion}

To promote a qualitative analysis and evaluation of the artefact, a focus group was created. The focus group participants were the authors of the article to explain the artefact and two external people. One is a professor and a master's in design thinking, marketing and innovation. In addtion to be a professor, he also has over 15 years of career in senior marketing positions in various sectors and countries. The other person is an expert in information systems architecture and a professor for more than 15 years in science and technology department of a university, be-sides having written many papers in the area of information systems, including CIS, and worked in different sectors in the IT area.

Was highlighted by the participants the importance of a system that can replicate the SCAMPER technique. All parties gave different perspectives on the system. These perspectives lead to an understanding that the artefact can be useful to different types of people. Then the participants focused on the practical aspects of the system discussing how the system could be used in practice while also providing some tips on how it can be use in marketing area.

The enthusiasm that the parties shown at this meeting allow us think that the proposal presented in this paper is relevant and can become an interesting functional system in the future.

\section{Conclusion}

The proposed conceptual model of an information system architecture for SCAMPER creativity technique based on CIS could be used as base for the design of a software for automatic ideas generation.

In the future, there is still much to be done. For instance, the system can be extended to a web-based crowdsourcing platform to be used in groups or in artificial intelligence systems with the ability to validate the different outcomes. Also, moving away from artificial intelligence, the system can be reformulated to have pre-defined contexts.

\section{References}

1. Adams, K., "The Sources of Innovation and Creativity.," Natl. Cent. 
Educ. Econ., 2005.

2. De Bono, E., Lateral Thinking, no. 416. Penguin Books, 1989.

3. Cross, N., Design thinking: understanding how designers think and work. Berg, 2011.

4. Young, M., Binning, C., and Young, M., Motivating people: using management agreements to conserve remnant vegetation;rft.aufirst. 1997.

5. Guilford J. P., Creative talents: Their nature, uses and development. Bearly limited, 1986.

6. Michalko, M., "Thinkertoys: A handbook of business creativity for the 90s," Long Range Plann., vol. 26, no. 3, p. 142, Jun. 1993.

7. Eberle, B., SCAMPER - Games for Imagination Development. Prufrock Press Inc, 1996.

8. Baecker, R. M., Readings in human-computer interaction : toward the year 2000. 1995.

9. Santos, V.; Mamede, H., "Creative Information Systems," in Encyclopedia of Internet Technologies and Applications, IGI Global, 2008, pp. 126-131.

10. Santos, V.; Mamede, H., "Uma Arquitectura para um Sistema de Informação Criativo," 2005.

11. Santos, V. Mamede, H., "Um Sistema de Informação Criativo baseado na técnica de criatividade whiteboard," 2006.

12. Santos, V. Pereira, J., Martins, J., Gonçalves, R., and Branco, F., "Creativity as a key ingredient of Information Systems," 2015.

13. Santos, V.; Mamede, H., and Goncalves, R., "Creativity technique Brute Thinking application using computer mediation," pp. 1-5, 2013.

14. Hevner, A. R. et al. (2004) 'Design Science In Information Systems Research', Design Science in IS Research MIS Quarterly, 28(1), pp. 75105.

15. Kuechler, B. and Vaishnavi, V. (2008) 'On theory development in design science research: anatomy of a research project', European Journal of Information Systems, 17(5), pp. 489-504. doi: 10.1057/ejis.2008.40. 